\title{
CLUSTERING ASPECTS IN WIRELESS SENSOR NETWORK:A REVIEW
}

Manisha Dhawan (Mtech Scholar) ${ }^{1}$, Amarpreet Singh (Associate professor \& head) ${ }^{2}$

\begin{abstract}
In wireless sensor networks, energy efficient clustering is an imperative research area which is used to extend the lifetime of the network. Various algorithms are developed to accumulate the power of sensor nodes and to enhance the network's ability. Clustering is a key tool that is very important in huge multi-hop wireless sensor networks for acquiring sociability, dipping power consumption's and augments the life time of network to accomplish better network concert. Continuing the research, this paper has focused on the energy efficiency in the network. Moreover, it explained the clustering protocol which are used to choose the clusters for the transmission of data from nodes to the cluster head and to the sink.

Keywords-Wireless Sensor Network, Clustering Protocols, Cluster Head, Sensor Nodes.
\end{abstract}

\section{INTRODUCTION}

A wireless sensor network (WSN) is rising in different areas consequently to provide novel occasions to the network as well as to the services [1]. WSN consists of miniature, battery powered sensor nodes that have inadequate storage, onboard processing and radio abilities. A sensor node consists of units that are explained below:

- A Sensing Unit: it is used to gather data from the surroundings.

- A Processing Unit: it is used for data processing.

- A Communication Unit: it is used to collect the data in the memory.

- A Power Unit: it is used to achieve the requisite tasks.

The data or report is intellect through the nodes and this report or data sent to the processing centre is named as "Sink".

[2] The major confront in the WSN is to expand the network lifetime by using their diminutive battery power.

The following ways are discussed that are important for utilize the energy perfectly:

- Deployment of sensor nodes.

- Energy Efficient Clustering.

- Energy Efficient Scheduling.

- Data Aggregation.

- Energy Efficient Routing Protocols.

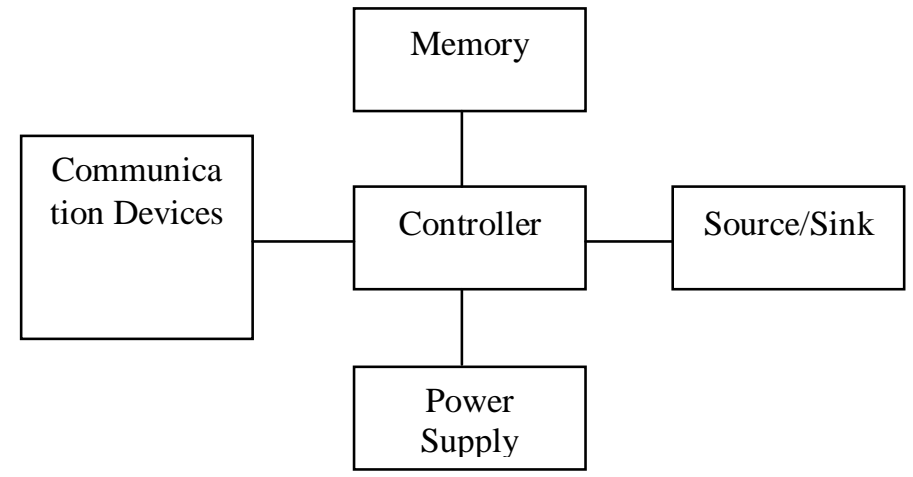

Fig 1: Overview of Sensor node Hardware components

Deployment of sensor nodes that can implemented in two traditions:

- Random Deployment

- Deterministic Deployment.

In the case of Random deployment, the nodes are disseminated randomly. In which Communication Devices, and the Memory Controller uses the Power Supply Source at higher rate. Since similar event can be takes place by using more than one node, results in high data redundancy as well as wastage of energy [3].Therefore to utilize the power in a professional way, the nodes are organized in Deterministic way. In Deterministic deployment, the network nodes are alienated into cluster. After the deployment, a Cluster Head $(\mathrm{CH})$ is chosen among each nodes that is being surrounded by the maximum energy [4]. The main motive of the Cluster Head is to gather data from the each nodes. At the end, the 
collected data are stored either to the Base Station (BS) or to the nearest Cluster Head. Well-organized energy utilization results shows that no redundancy of data as well the wastage of energy takes place.

\section{SOME COMMON ISSUES IN DESIGNING THE CLUSTERING PROTOCOLS:}

Following are the common issues that occurs at the time of designing the clustering protocols [5]:

- Fault Tolerance: The WSN consists the sensor nodes that may fail due to the lack of energy. Fault tolerance can be defined as the capability of the sensor node in which the routine or functionality of the network should not be exaggerated if the malfunction of node takes place.

- Operating Environment: The main features of the WSN like constancy, Lifetime, Throughput fluctuations that changes with respect to the operating environment.

- Power Consumption: In WSN, the main purpose of the network should be to utilize the well-organized energy that will work as the supplement for its network lifetime.

- Data Aggregation: when the data is collected from two or more data sources it is known as the data aggregation. In the WSN, data is produced through diverse sources. If the data is collected firstly and then it is sent to the base station, Its becomes beneficial as lot of energy is saved. The process of data aggregation helps to reduce the data redundancy of the network which increases the efficiency of the network.

- Quality of Service: The selection of the routing protocols that plays an imperative role in the quality of service of the network. The quality of service is deliberate in terms of length of time, power competence, collaborationprocessing and data consistency.

- Data Latency and Overhead: Occasionally data aggregation possibly results in data latency and even overhead the network.

- Node Deployment: The node deployment plays an imperative role for the competent energy utilization. Because the random deployment condenses the competence of the network through the wasting of energy where as deterministic deployment technique helps the network to augment its lifetime by competently utilize the energy.

\section{EXISTING PROTOCOLS}

For the well-organized energy utilization, many clustering protocols are proposed some of them are discussed below:

\section{a) LEACH}

Low Energy Adaptive Clustering Hierarchy("LEACH"), it is a hierarchical protocol in which the data is aggregated and compressed [6]. After that data is sent to the "Sink"(Base Station), where every node broadcast the data to their cluster head. At every round, a cluster head is selected and is determined through a stochastic algorithm. This protocol uses two types of phases as:

- $\quad$ set up phase

- steady state phase.

It is imperative to note that, when a node that has previously become cluster head is not be able to become cluster head for the subsequently $1 / \mathrm{P}$ round wherever $\mathrm{P}$ is the probability to become cluster head. Every node that are not become the cluster head joins their nearest cluster head and selects a schedule for that exacting node so as to convey the data [7]. The TDMA (Time Division Multiple Access) id the schedule that is used for the communication with cluster head. The problem of LEACH protocol is that the distribution of nodes in clusters is completed in an arbitrary fashion [8]. Its division is also done in non-uniform fashion which decreases the network lifetime as well as the competence of the network.

\section{Advantages}

1. It is widely used in WSN as hierarchical routing and it is easy to appreciate.

2. In this Equal Load sharing is performed, Because when a node is chosen as a Cluster head that can't be a cluster head again in the next round.

3. It is cooperative to improve the lifetime of the network .

4. It is suitable to the average energy consumption.

\section{$>$ Disadvantages}

1. Due to its characteristic that is arbitrary assortment of clusters heads, it concerns the network robustness. Since in this the probability of a node to become a cluster head is more or equal in every round irrespective to its enduring energy [9]. Therefore if the node is selected with the lowest energy as the cluster head then it can consequence or decrease the lifetime of the network. Since the node with the minimum amount of vigor will pass away quickly as contrast to other nodes.

2. The randomization of cluster head escorts to the imbalanced distribution which enlarges the energy consumption and abscond a negative contact on the all over concert of the network.

3. In this the cluster heads unswervingly communicate to the sink node. It revenue that the cluster heads which are situated at the aloofness from the sink node also have to communicate directly to the sink node which results shown that more energy consumption. Therefore the far situated cluster heads will collide earlier which reduces the recital of the network. 


\section{b) HEED}

HEED stands for Hybrid Energy-Efficient Distributed Clustering. In HEED does not surround any communication overhead so it did not ease the competent distribution of cluster head nodes over the network. LEACH-C protocol is use to conquer this problem, it is a centralized technique but scalable to imperfect number of sensors only [10]. Various clustering algorithms are obtainable which produces the additional uniform clusters at the outlay of overhead in cluster configuration. One of the techniques is HEED which is used for distributed algorithms that are simple and rapid to exchange [11]. It uses a cluster formation algorithm, in which every node is dispensed with a cluster head probability which is the function of their remaining energy. Cluster head probability is used to choose that whether the sensor node is applicant for cluster head for this encircling or not.

\section{$>$ Advantages}

Following are the advantage of HEED :

1. In this, no need to comprise the comprehensive knowledge concerning the nodes in a network.

2. It concludes the dispensation at $\mathrm{O}$ (1) round or iteration,

3. It considers that every node is part of a solitary cluster heads.

\section{$>$ Disadvantages}

1. Produce supplementary cluster heads, which are inappropriate or have no necessitate to produce these cluster heads. Creation of inappropriate cluster heads escorts to the disproportion in the level of power consumption.

2. appropriate to the dissipation of energy the routine of the network gets exaggerated. .

3. In order to organize the cluster heads. HEED needs huge number of iterations and during each and every iteration lot of packets are transmitted.

4. The cluster heads situated close to the sink node tired earlier since it has large exertion load.

\section{c) TEEN}

TEEN stands for Threshold sensitive Energy Efficient sensor Network protocol and it is used for reactive networks. It is an appliance that is used for senses the temperature. It is an additional energy well-organized protocol as contrast to the conventional protocols [12]. To overcome the restraints of LEACH protocol, the TEEN protocol is developed. It is enhanced version of LEACH protocol. TEEN is not suitable for huge scale networks because it lacks:

- Before the events happened, the cluster-heads choosing arbitrarily. It makes the sensors out of the event region collecting into clusters and transmitting data, causing redundant power consumption and unhinge clusters.

- $\quad$ Selecting the cluster-heads without considering their remaining energy. It may select the sensors with less power as the cluster-heads and then origin them untimely death.

- The cluster-heads broadcast data to the sink node unswervingly. Thus one-hop transmission mode may result in the cluster-heads far away from the sink node rapidly dead.

\section{$>$ Advantages}

1. It decreases the amount of power that is used for transmission of the data by transmitting the necessary or responsive data only.

2. It results shown the enhancement in received data and competence of the network.

3. It has the characteristic of forcefulness.

\section{$>$ Disadvantages}

1. The entire process relies on the threshold because it is a threshold sensitive protocol, therefore this protocol is not appropriate for periodic report applications.

2. In TEEN the information is transmitted only at the cluster heads. So, in case if it is not able to discover the cluster heads then the data is cracked.

\section{d) DEEC}

DEEC stands for distributed energy efficient clustering that is used for heterogeneous wireless sensor network. In this protocol the ratio between the remaining energy on every node and average energy on the network is intended. Its probability ratio is used to choose the cluster-heads [13]. The number of rotation on every node varies along with the variations in preliminary and remaining energy i.e. DEEC transforms the rotation on every node into energy. The nodes which have lofty amount of residual energy and high preliminary energy is much appropriate for cluster head candidate node as contrast to the nodes with low energy. Therefore DEEC can expand the lifetime of the network with the use of heterogeneous aware clustering algorithms. DEEC can increase more effective messages as contrast to the classical clustering algorithms. DEEC is more apposite for Multi-level heterogeneous networks.

\section{$>$ Advantages}

Following are the advantages of DEEC:

1. Unlike other protocol there is no necessitate of having the information of energy of rest of the nodes in each and every round.

2. DEEC is most appropriate protocol for multi-level heterogeneous wireless networks.

\section{$>$ Disadvantages}

1. Advance nodes are reprimand in DEEC, because when the energy of the advance nodes get tired then these fall under the category of normal nodes. Therefore the advance nodes die quickly as contrast to other nodes in the network. 


\section{e) PEGASIS}

PEGASIS stands for Power-Efficient Gathering in Sensor Information System that is a chain-based power effective algorithm [14]. It is based on subsequent two parameters:

- Chaining

- Data fusion

It vocations similarly LEACH. In PEGASIS, every node can act as a leader of the chain and chain is also created with the help of greedy algorithm and can install with the use of sensor nodes. It is based on following assumptions:

- Sensor nodes have comprehensive knowledge of the network

- $\quad$ Every nodes are stationary

- Nodes have knowledge about the position of all other nodes.

It also countenance some problems as LEACH suffers. One of the main disadvantages of PEGASIS is that it is not scalable so it cannot be used in case of wireless sensor networks due to the lack of knowledge concerning the nodes because of huge number of nodes.

Advantages

1. PEGASIS is an superior version of LEACH protocol.

2. In this protocol the energy is distributed uniformly to every sensor nodes so that the nodes can remain in working condition for the extensive period.

\section{$>$ Disadvantages}

The disadvantage of the PEGASIS is :

1. The lifetime of the sensor node is diminutive.

2. Because the nodes have to directly communicate the sink node which leads to the utmost energy consumption for the nodes which are situated far away from the sink node.

\section{f) SEP}

SEP is a protocol which supports the assortment in two levels of network. Here assortment refers to the preliminary energy allotment to the sensor nodes [15]. According to the assumptions of SEP protocol in genuine time network, there are two types of energy and hence it defines the nodes as proceed nodes and usual nodes. The only difference between proceed nodes and usual nodes is that the proceed nodes have additional amount of energy as contrasts to the usual nodes. On the basis of initial energy it assigns the prejudiced probability to nodes. It also overcomes the difficulty of cluster formation which exists in LEACH protocol.

\section{$>$ Advantages}

The advantage of SEP is that it doesn't necessitate the information concerning the energy level of nodes at each election round.

\section{$>$ Disadvantages}

The disadvantage of the SEP is that in this there are two types of nodes in the network which leads to the clashes while cluster head selection. In this cluster head selection is not dynamic. Therefore the resultant that the normal nodes will tire first.

\section{RELATED WORK}

Various algorithms had been proposed to choose an appropriate cluster head for clusters through several researchers. The authors recommend that these algorithms were mainly use in wireless sensor networks where there was data aggregation and energy utilization efficiently.

Mrs. Praia Sari, et al [1] In current enlargement, the use of Wireless Sensor Network (WSN) to grip more composite functions was rising drastically which in turns to needs their powered battery sensors to competently utilize their energy in order to prolong the network lifetime for the heterogeneous nodes. Clustering is one of the approach that is used to optimize this energy consumption and increasing the network lifetime as well. In topical years, various clustering protocols had been proposed based upon heterogeneity like SEP,LEACH,E-LEACH etc. In these protocols, a Cluster Head $(\mathrm{CH})$ was chosen having maximum energy among all the nodes. In this paper to study several clustering protocols that was suitable for heterogeneous wireless sensor network.

Rashid Raman Shoo, et al [2]due to energy constraint of sensor nodes, scalability and prolonging the network life time were the crucial matter for wireless sensor network designer In this paper, the author presented a trust based protected and energy competent clustering algorithm in wireless sensor network (TREE-CR). The proposed energy competent and trusted clustering algorithm can defend the wireless sensor network from several type of malicious nodes. Moreover, in TREE-CR the author had also proposed a sensible energy consumption model for precise prediction of network life time and this algorithm can also sensed the malicious nodes in the network. This energy consumption model considers all the fundamental actions of a sensor node. Furthermore, the author had also compared this proposed algorithm TREE-CR with most accepted protocol LEACH. 
Sneak Kemble et al, [3] proposed a system to conquer the cons on the preceding papers i.e. to discover the harassed node as well as cluster head. The results established the network life as well as correctness of the network was augmented as well as keeping away harasser from the system through making the use of proposed system.

Sango Mondale, et al, [4] proposed an energy well-organized load balanced data collecting protocol coined as RFLEACH where partitioning was done by using rough fuzzy c means (RFCM) and cluster head assortment was based upon fuzzy logic. Simulation results indicated that RF-LEACH achieved better than LEACH, FCM LEACH and Fuzzy LEACH in terms of expanding the network lifetime and throughput in a load balanced way. The results were shown to be statistically noteworthy.

Anita Chauvin, et al, [5] proposed a clustering routing protocol CRT2FLACO on the basis of type-2 fuzzy logic as well as ACO for balancing the network load and to enhance the lifetime of WSN. Our proposed protocol determines the three fundamental parts of residual energy, neighbor nodes as well as the distance to base station as inputs of a type-2 Madman FLS and after that calculate the possibility of a node upgrading as a candidate cluster head and the cluster head competition radius. Implementation of type-2 fuzzy logic is better way to control the overhead existing in WSN. Load of network is efficiently handled by fuzzy unequal competition radius as well as implementation of multi-hop routing protocol technique for data transmission. Additionally, security of data is determined by utilizing encryption algorithm in transmission of data. Network lifetime is enhanced due to system has high packet delivery rates.

K V Praveen Kumar, et al, [6] The superfluous data compilation in dense network results in additional energy consumption. DRINA stands for Data Routing In-Network Aggregation was one of the suitable algorithm that was proposed to decrease energy consumption in dense network environment through minimizing the number of communications from source to sink. At this point the Data transmission was carried out by using data aggregation in cluster based environment with the use of shortest path approach. But due to incompetent cluster head assortment technique, it was not capable to manage cluster head disappointments and avoid the energy drain in sensor nodes along the common static path that leads network partition. In order to defeat these problems the author was proposed an algorithm known as Energy Efficient Routing using Dynamic Cluster approach (EBDRA), that comprised the efficient cluster head selection approach and dynamic route selection for the dependable data transmission. In this approach the cluster head selection technique engages an efficient scheme of cluster head selection. The proposed algorithm decreases the communication overhead, power consumption and enhanced the network lifetime.

\section{CONCLUSION AND FUTURESCOPE}

The above study is concluded that well-organized power utilization plays an imperative role in the wireless sensor network. The protocols like LEACH, SEP, HEED etc that are used for dividing the network into cluster and selects a cluster head $(\mathrm{CH})$ among that node have maximum power among all other nodes. But a lot of power is exhausted in transmit which decreases the competence of the network. Therefore, a new technique can be proposed in order to select a cluster head on the basis of such parameters which can reduce the consumption of energy and enhanced lifetime of the network.

\section{REFERENCES}

[1] Mrs Priya Suri et al, "Review Paper on Various Clustering Protocols Used In Wireless Sensor Network (WSN)", IEEE, 2015.

[2] Rashmi Ranjan Sahoo, et al, “Tree-Cr: Trust Based Secure And Energy Efficient Clustering In Wsn”, Ieee , Pp: 5325-538, 2013.

[3] Sneha Kamble, et al, "Reliable Routing Data Aggregation using Efficient Clustering in WSN" IEEE, Pp: 246-250,2016.

[4] Sanjoy Mondal, et al, "Energy Efficient Rough Fuzzy Set based Clustering and Cluster Head Selection for WSN", IEEE, Pp:439-444, 2016.

[5] Anita Chavan, et al, "Securely energy aware routing in WSN with efficient clustering", IEEE, Pp: 624-628, 2016.

[6] K. V. ;Praveen Kumar, et al, "EBDRA: Energy balanced dynamic cluster routing approach for WSN”, IEEE, Pp: 141-145,2016.

[7] Dan Liu, et al, "Cluster based energy efficient transmission using a new hybrid compressed sensing in WSN", IEEE, Pp:372-376, 2016.

[8] Mihaela I. Chidean, et al, "Scalable Data Coupled Clustering for Large Scale WSN”, IEEE, Pp:4681-4694, 2015.

[9] Pawan Singh Mehra, et al, "Energy efficient self Organising Load Balanced Clustering Scheme for Heterogeneous", IEEE, Pp:1 -6, 2015

[10] Soumita Sen, et al, "Design of Cluster Chain based WSN for energy efficiency", IEEE, Pp:150-154, 2016.

[11] Firoj Ahamad et al, "Energy Efficient Region based Clustering Algorithm for WSN using Fuzzy Logic", IEEE, Pp:1020-1024, 2016.

[12] P. M. Harsha, et al, "Network Lifetime Enhancement of Clustering Approach using Handoff Mechanism in WSN", IEEE, Pp:809-812, 2016.

[13] N. Swathi, et al, "Zone based hierarchical energy efficient clustering scheme for WSN", IEEE , Pp:136-140, 2016.

[14] Mei Wu, et al, "Lifetime Enhancement by Cluster Head Evolutionary Energy Efficient Routing Model for WSN", IEEE, Pp:545-548,2016.

[15] Muneer Bani Yassen, et al, "Secure Low Energy Adaptive Clustering Hierarchal based on the internet of things for wireless sensor network(WSN): Survey", IEEE, Pp:1-9, 2016.

[16] Yu Xiuwu, et al, "Wsn Monitoring area Partition Clustering Algorithm for Energy Balanced", IEEE, Pp:80-84, 2016.

[17] R. Giridhar, et al, "Cluster based Certificate Authority Scheme in WSN, "IEEE, Pp:367-371, 2016.

[18] Xiang Gao, et al, "Coverage of Communication based sensor nodes Deployed location and energy efficient clustering algorithm in WSN", IEEE, Pp:698-704, 2010.

[19] Haixia Peng, et al, "Toward Energy Efficient and Robust Large-Scale WSN: A scale free Network Approach", Pp:4035-4047, 2016.

[20] Zhoxon Liu, et al, "An approach of Distributed joint optimization for cluster based wireless Sensor Networks", IEEE, Pp:267-273, 2015.

[21] Duc Chinh Hoang, et al, "Realisation of a Cluster based Protocol using Fuzzy C-means Algorithm for Wireless Sensor Networks", IEEE, Pp:163-171, 2013.

[22] Zhezhuang $\mathrm{Xu}$, et al, "Joint Clustering and Routing Desgin for Reliable and Efficient DatavCollection in Large Scale Wireless Sensor Networks", IEEE, Pp:520-532, 2016.

[23] Sunil Kumar Singh, et al, “A Survey on Successors ofLEACH Protocol”, IEEE, Pp:4298-4328, 2017

[24] Anindita Ray, e al, "Energy Efficient Clustering Protocol based on K-means(EECPK- means)- midpoint algorithm for enhanced network lifetime in wireless sensor network", IEEE, Pp:181-191, 2016. 
[25] Trong Thua Huynh, et al, "Delay Constrained Energy Efficient Cluster based multi-hop routing in wireless sensor networks”, IEEE, Pp:580-588, 2016

[26] Mihaela I. Chiden, et al, "Energy Efficiency and Quality of Data Reconstruction Through Data-Coupled Clustering for Self Organized Large Scale WSNs” IEEE, Pp:5010-5020, 2016

[27] Oualid Demigha, et al, "On Energy Efficiency in Collaborative Target Tracking in Wireless Sensor Network: A Review”, IEEE, Pp:1210-1222, 2013.

[28] Majdi Mansouri, et al "Secure and Robust Clustering for Quantized target tracking in wireless sensor networks", IEEE, Pp:164-172, 2013.

[29] Wenliang Wu, et al, "Improved Clustering algorithm based on energy consumption in wireless sensor networks", IEEE, Pp:47-53, 2017.

[30] Jin Shyan Lee, et al, “An improved Three- layer Low- energy Adaptive Clustering Hierarchy for Wireless Sensor Networks", IEEE, Pp:951-958, 2016. 